\title{
Antimicrobial susceptibility pattern of urinary tract isolates of citrobacter species in a tertiary care hospital
}

\author{
Shobha K.L ${ }^{1 *}$, Akshatha S.J. ${ }^{2}$, Amin Sonam G.S. ${ }^{2}$, Ramachandra $^{3}{ }^{3}$ \\ ${ }^{1}$ Department of Microbiology, Melaka Manipal Medical College(Manipal Campus), ManipalUniversity, Manipal- 576104, \\ Udupi District, Karnataka, India \\ ${ }^{2}$ Department of Microbiology Kasturba Medical College, Manipal,Manipal University, Manipal- 576104, \\ Udupi District, Karnataka, India \\ ${ }^{3}$ Department of Surgery, Kasturba Medical College,Manipal,Manipal University,Manipal- 576104,Udupi District,Karnataka,India \\ *Corresponding author E-mail: shobhamicro@yahoo.com
}

\begin{abstract}
Introduction: The genus Citrobacter is a distinct group of aerobic Gram-negative bacilli from the Enterobacteriaceae family. It is an opportunistic pathogen that can cause diarrhea, septicemia, meningitis, urinary tract and respiratory system infection. Its isolation in the hospital settings is increasing. It is a present challenge to the clinical microbiologist because of their increased occurrence in nosocomial infection.

Objectives: The current study was done to find out the prevalence and antibiotic susceptibility pattern of Citrobacter species from urinary tract isolates.

Materials and Methods: Urine samples received from January 2013 to December 2013 were considered. A total of 9186 urine samples were considered, out of which 6381 were sterile and 2805 plates had growth.65(2.312\%)of the growth plates had Citrobacter species growth. Citrobacter species were identified by conventional biochemical method. Antibiotic sensitivity test was performed by KirbyBauer Disc diffusion method.

Results: Out of 65 patients female: male ratio was 17:15.5.The age groups were 0-9years; 8 patients(12.3\%),10-19years;3 patients(4.615\%),20-29years;11patients (16.9\%),30-39years;6patients(9.28\%),40-49years;12 patients(18.46\%),50-

59 years;5patients $(7.69 \%), \geq 60$ years; 20 patients $(30.76 \%)$. The effective agent against Citrobacter species was found to be Imipenem (90.76\%) then Cefoperazone-Sulbactum (89.23\%) followed by Piperacillin-Tazobactam and Amikacin (87.69\%).6 Multidrug resistant strains and 8 were ESBL producers.

Conclusion: From the study it was seen that multidrug resistant strains are emerging. Proper surveillance in the antimicrobial sensitivity of Citrobacter was required. The age group 60 and above was found in majority to have culture positive Citrobacter spp. Depending on antibiotic sensitivity pattern of Citrobacter isolates, the antibiotics should be used. Proper infection control measures should be taken to prevent the spread of the pathogen.
\end{abstract}

Keywords: Antibiotic Susceptibility, Citrobacter Spp, Multi-Drug Resistant Bacteria, Urinary Tract Infections.

\section{Introduction}

Urinary tract infection (UTI) continues to be the commonest nosocomial infection according for approximately $40 \%$ of all hospital acquired infections and it is one of the most important causes of morbidity and mortality ( Basavaraj C et al; 2013). Urinary tract infection (UTI) is the third most common infection experienced by humans after respiratory and gastro-intestinal infections. UTI may be defined as a condition in which bacteria are established and multiplying within the urinary tract. Diagnosis requires demonstration of bacteriuria. Exceptions to this include patients with pyogenic abscess of kidney or perinephric tissue, obstructed pyelonephritis or bacterial prostatitis in whom the urine may be sterile (Barton et al; 1982).

The genus Citrobacter is a distinct group of aerobic Gramnegative bacilli from the Enterobacteriaceae family (Najar M.S et al; 2009). It is an opportunistic pathogen that can cause diarrhea, septicemia, meningitis, and urinary tract and respiratory system infection (Metri BC et al; 2011). Citrobacter isolates were found to be the third most common organism causing UTI in hospitalized patients after Escherichia coli and Klebsiella species accounting to $9.4 \%$ of all isolates (Lipsky BA et al; 1980). It is a present challenge to the clinical microbiologist because of their increased occurrence in nosocomial infection.

The aim of the present study was to find out of prevalence and antibiotic sensitivity pattern of Citrobacter spp in patients admitted to or attending outpatient Departments with history of urinary tract infections in a tertiary care hospital.

\section{Materials and methods}

Urine samples received from January 2013 to December 2013 from Kasturba hospital, Manipal were included in the study. A total of 9186 urine samples were included in the study, out of which 6381 were sterile and 2805 plates had growth. Identification was by done using standard microbiological techniques .( Murray PR et al ;2005). 
Antibiotic susceptibility test was performed by Kirby-Bauer Disc diffusion method. Test strains were pre-incubated in peptone water at $37^{\circ} \mathrm{C}$ at an optical density of $0.5 \mathrm{Mc}$ Farland standard. This suspension was used to inoculate the strains onto the Muller Hinton agar plate by swabbing them with a sterile cotton swab and performing lawn culture as recommended by clinical and laboratory standards institute (Wayne,PA ;2005)

The antibiotic discs used for sensitivity testing were obtained from $\mathrm{Hi}$-Media,Mumbai,India and the following antibiotics were used. Amikacin $(30 \mu \mathrm{g})$, Ampicillin $(10 \mu \mathrm{g})$,Ceftriaxone $(30$ $\mu \mathrm{g})$,Amoxicillin-Clavulanic acid(20/10 $\mu \mathrm{g})$ Cefuroxime(30 $\mu \mathrm{g})$,Cotrimoxazole $(1.25 \mu \mathrm{g} / 23.75 \mu \mathrm{g})$, Gentamicin(10 $\mu \mathrm{g})$,
Netilmicin(30 $\mu \mathrm{g})$,Norfloxacin(10 $\mu \mathrm{g})$, Aztreonam(30 $\mu \mathrm{g})$,Cefoperazone-Sulbactam(75/30 $\mu \mathrm{g})$,Cefipime(30

$\mu \mathrm{g})$, Meropenem $(10 \mu \mathrm{g})$ and Piperacillin-Tazobactam(100/10 $\mu \mathrm{g})$. $65(2.312 \%)$ of the growth plates had Citrobacter species growth.

\section{Results}

Out of 160 patients 34 were females $(52.3 \%)$ and 31 were males (47.69\%) (Fig1).

\section{Prevalence of Citrobacter species based on gender}

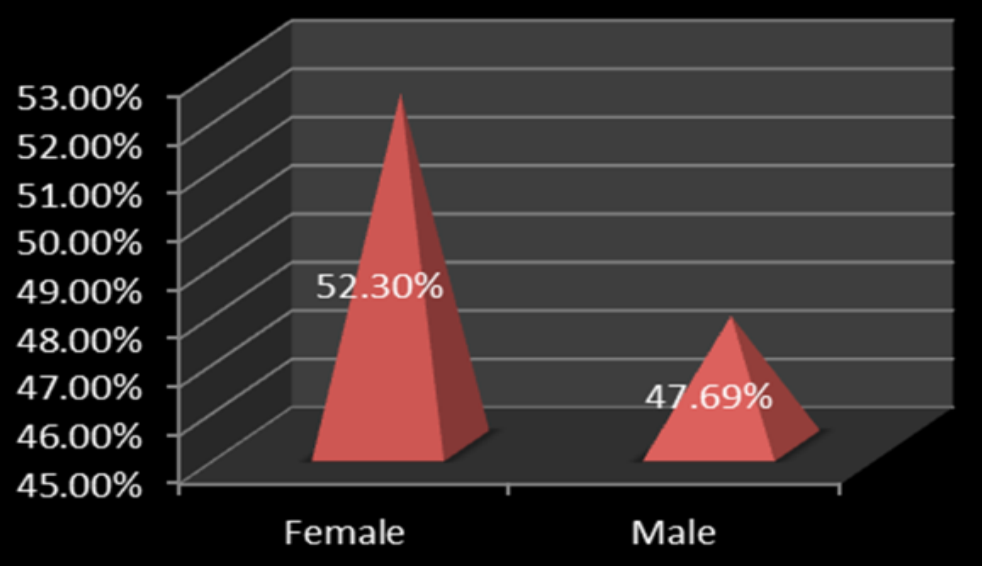

\section{- Prevalence of Citrobacter species based on gender}

Total $=65$ Citrobacter species Females $34(52.3 \%)$ Males=31(47.69\%)

Fig. 1: Prevalence of Citrobacter Species Based on Gender

\section{Prevalence of Citrobacter species based on age group}

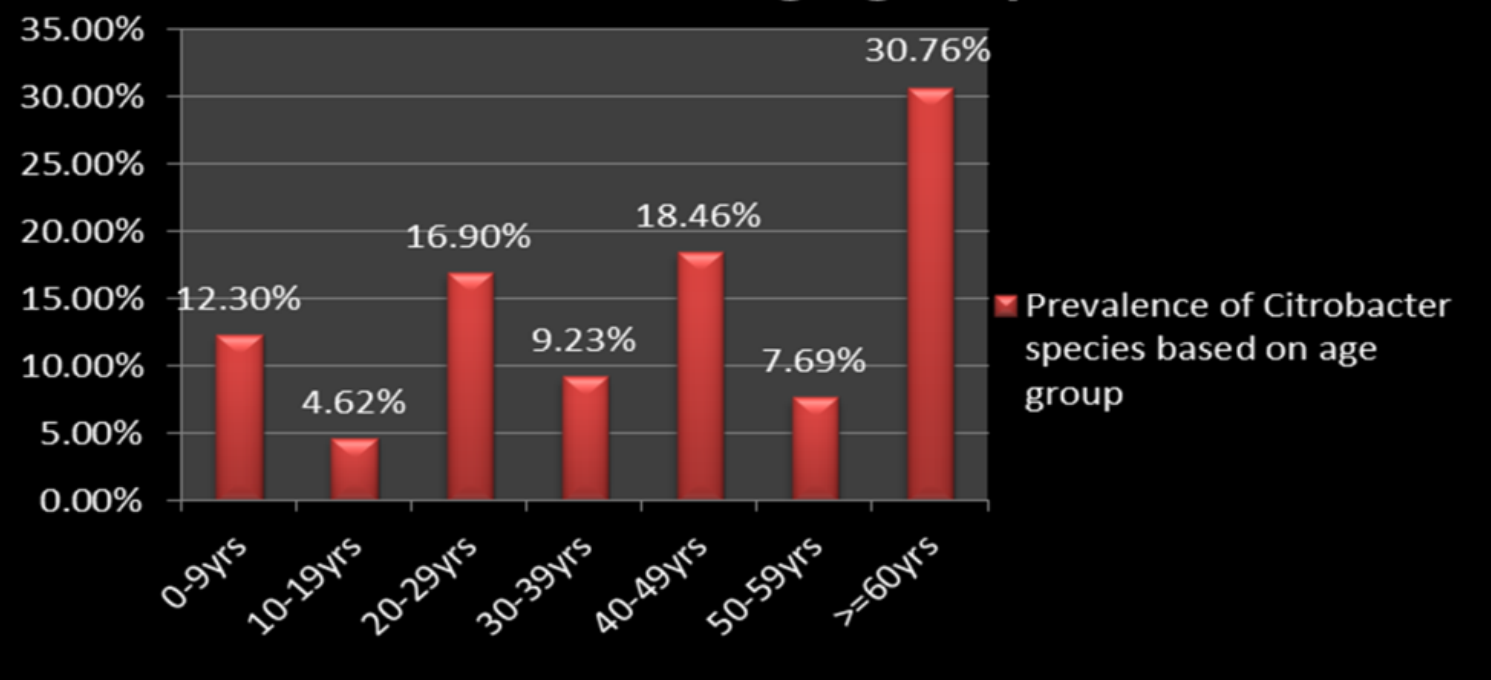

Fig. 2: Prevalence of Citrobacter Species Based on Age Groups 


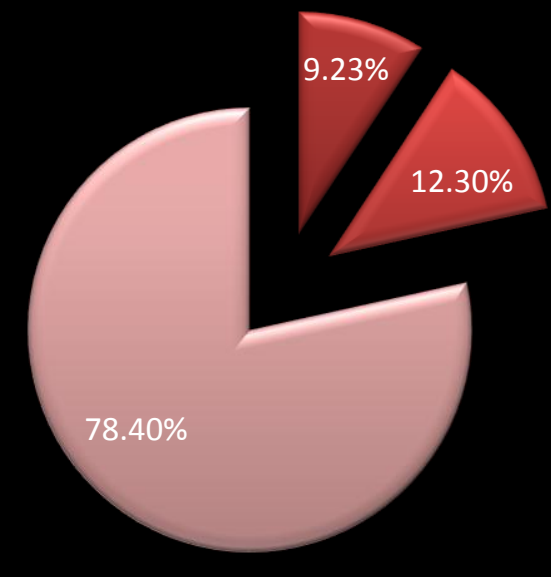

₹ MDR Strains

- ESBL producers

Mensitive strains

Fig. 3: Pie Chart was Showing Percentage of Sensitive and Resistant Strains.

\section{Number of patients}

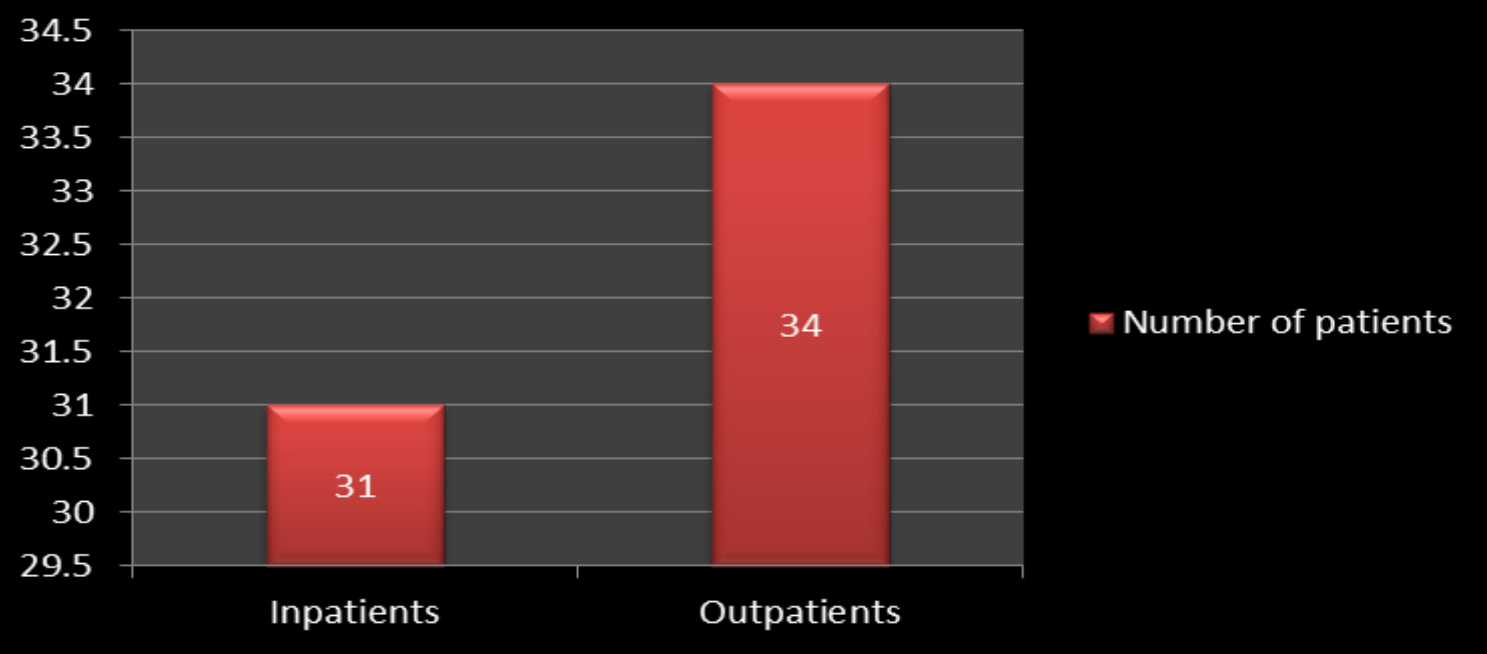

Fig. 4: Characterization of Patients Based on Number of Inpatients and Outpatients.

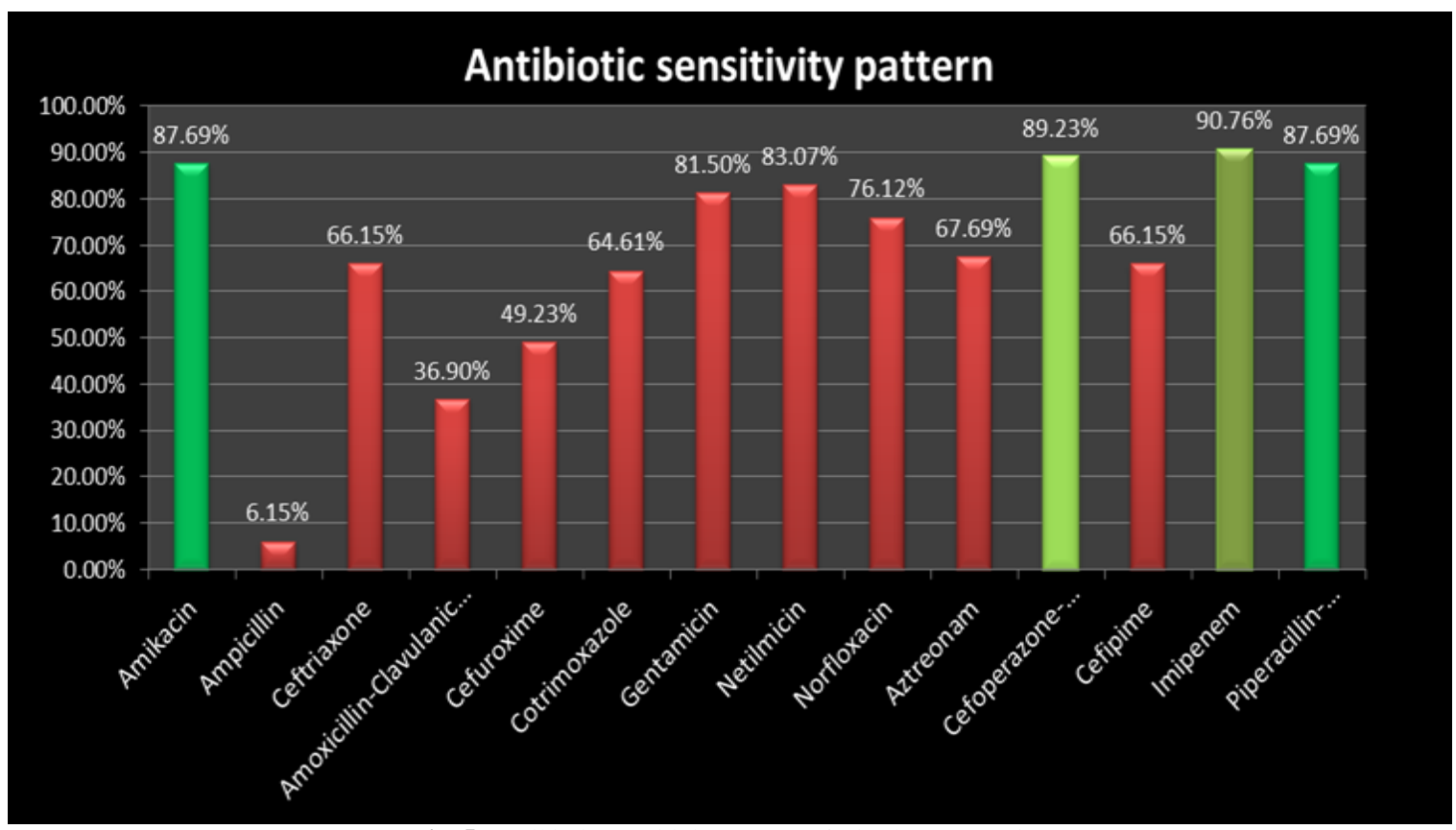

Fig. 5: Antibiotic Sensitivity Pattern of Citrobacter Species 
The age groups were 0-9years; 8 patients(12.3\%),10-19years; 3patients(4.615\%), 20-29years;11patients (16.9\%),3039years;6patients(9.28\%),40-49years; 12 patients(18.46\%),5059years;5patients(7.69\%), $\geq 60$ years;20 patients(30.76\%)(Fig:-2). Out of the 65 strains 14 were found to be Multidrug resistant and 8 of which were ESBL producers. (Fig:-3) MDR (Multi drug resistant), ESBL (Extended spectrum beta lactamase).

The data was also studied under the parameter of inpatient and outpatient entry. 31 patients were inpatients and 34 were out patients. (Fig4).

Depending upon the conventional biochemical method Citrobacter was speciated. Antibiotic sensitivity pattern of the following Citrobacter was found to be as follows.

\section{Discussion}

It was observed in other study that females were more prone to urinary tract than males, females $34(52.30 \%)$ and males 31(47.69\%). Similar study conducted by (MY Tula et al; 2014) showed that females were suffering more than males in urinary tract infection. Citrobacter were emerging pathogens causing Urinary tract infection. Citrobacter was the leading cause of urinary tract infection (Samonis et al; 1991). There had been increase in the isolation of Citrobacter species $(2.312 \%)$ when compared with the other similar study which was $0.8 \%$ (Rong Zhang et al; 2008) .In our study we found that Citrobacter species isolates were almost equal in female patients and male in contrast to other study (Basavaraj et al; 2013). Earlier studies in the same hospital done by (Shobha K.L et al; 2007) had two strains out of ten strains producing ESBL. In the present study there were eight strains out of $65(12.30 \%)$. This showed that there was an increase in the ESBL strains of citrobacter species.Buddha Bahadur Basnet et al ;2013, in their study showed that all the citrobacter species isolated from urine species were multidrug resistant strains. In our study 8 strains out of 65 were multidrug resistant strains(12.90\%).In a study conducted by ( Ravindranath Misra et al;2012) reported $44.73 \%$ ESBL producers and had only four strains out of 114 multidrug resistant strains.

Susceptibility to Amikacin was $87.69 \%$ but study conducted by (Gandam Pavani; 2012) showed $100 \%$ susceptibility to Amikacin in contrast (K Ashish et al; 2012) showed only $25 \%$ susceptibility to Amikacin. Most effective agent against Citrobacter species was found to be Imipenem $(90.76 \%$ sensitivity) followed by Cefoperazone-Sulbactam $(89.23 \%$ sensitivite $)$ when compared with other study where most effective drug was found to be Imipenem (91.8\% sensitive) followed by Piperacillin-Tazobactam (58.3\% sensitive) (Metri BC et al;2011). To conclude, we would like to bring notice to the fact that Citrobacter though not uncommon isolate is increasing with its multi-drug resistance and Extended Beta -lactamase producers. Proper surveillance in the antimicrobial sensitivity of Citrobacter is required. Depending on antibiotic sensitivity pattern of Citrobacter isolates the antibiotics should be used judiciously. Infection control measures should be taken to prevent the spread of the pathogen.

\section{References}

[1] Basavaraj C. Metri, P. Jyothi, Basavaraj V. Peerapur. Antibiotic resistance in Citrobacter spp. isolated from urinary tract infection. Urology Annals Oct - Dec 2013 Vol 5 Issue 4

[2] Barton LL, Walentik CW;Citrobacter diversus urinary tract infection,Am J Dis Child.1982;136;467-468.

[3] Najar M.S, Saldanha C.L., and Banday K.A. Approach to urinary tract infections. Indian J Nephrol. Oct 2009; 19(4): 129-139. http://dx.doi.org/10.4103/0971-4065.59333.

[4] Metri BC, Jyothi P, Peerapur BV. Anti-microbial resistance profile of Citrobacter species in a tertiary care hospital of southern India. Indian J Med Sci 2011; 65:429-35 http://dx.doi.org/10.4103/00195359.109259 .
[5] Lipsky BA,Hook EW, 3rd Smith AA, Plorde JJ.Citrobacter infection in humans; experience at Seattle veterans Administration Medical Centre and review of literature. Rev Infect Dis.1980; 2; 746 - 760. http://dx.doi.org/10.1093/clinids/2.5.746.

[6] Murray PR, Holmes B,Auken HM. Citrobacter, Enterobacter, Klebsiella, Plesiomonas, Serratia and other members of the enterobacteriaceae In;Borriello SP,Murray PR, Funke G, editors. Topley and Wilson's Microbiology and Microbial infections. 10TH ed. London; Hodder Arnold;2005;pp 1474- 506

[7] Wayne, PA, CLSI document; 2005, Clinical and Laboratory standards Institute Performance standards for antimicrobial susceptibility testing.Fifteenth informational supplement; pp M 100 - S 15.

[8] MY Tula and O.Lyoha; Distribution and Antibiotic susceptibility pattern of bacterial pathogens causing urinary tract infection in Mubi general hospital-Yola, Nigeria; British journal of Medicine and Medical research; 2014; 4(19); 3591- 3602

[9] Samonis G,Anaissie E,Elting L,Bodey GP; Review of Citrobacter bacteremia in cancer patients over a sixteen year period.Eur J Clin Microbiol Infect Dis; 1991;10;479 - 485) http://dx.doi.org/10.1007/BF01963933.

[10]Rong Zhang, Lijiang Yang, Jia Chang Cai, Hong Wei Zhou and Gong-Xiang Chen. High-level carbapenem resistance in a Citrobacter freundii clinical isolate is due to a combination of KPC-2 production and decreased porin expression. Journal of Medical Microbiology (2008), 57, 332-337 http://dx.doi.org/10.1099/jmm.0.47576-0.

[11] Shobha K.L,Gowrish Rao S, Sugandhi Rao, Sreeja C.K ; Prevalance of Extended spectrum Beta-Lactamase in Urinary isolates of Escherichia coli,Klebsiella and Citrobacter species and their antimicrobial susceptibility pattern in a tertiary care hospital;Indian Journal for the practicing Doctor; vol 3,No 6 (2007 -01 - 2007 -02).

[12]Buddha Bahadur Basnet, Kumananda Acharya, Santosh Khanal, Rajan Kumar Dahal;Trends in Antimicrobial resistance among common isolates of urinary tract infection in tertiary care hospital of Nepal; International research journal of pharmaceutical and applied sciences; 2013;3(4); 22-26.

[13]Rabindranath Misra, Nageswari Gundham, Moumita Sardar,Mahadev Ujagare,Kalpana Ungadi,Chanda Vyawahare,Indranath Roy and Savitha Jadav;High prevalence of multidrug resistant citrobacter spp from tertiary care hospital,Pimpri, Pune,India;Journal of Pharmaceutical and biomedical sciences;2012;Dec 25(25),158 - 163.

[14]Gandam Pavani; Drug susceptibility pattern of Klebseilla and citrobacter infections in India; Jounal of Microbiology and biotechnology Research; 2012; 2(4); 619- 620.

[15]K Ashish,S Nachatar,A Aruna;Journal of Clinical and Diagnostic research;2012;6(4);642-644. 